\title{
Editorial
}

\section{Nanomaterials and Nanodevices for Energy Applications}

\author{
Wenjie Mai, ${ }^{1}$ Yu-Lun Chueh, ${ }^{2}$ Christian Falconi, ${ }^{3}$ and Matthew T. Cole ${ }^{4}$ \\ ${ }^{1}$ Department of Physics, Jinan University, Guangzhou 510632, China \\ ${ }^{2}$ Department of Materials Science and Engineering, National Tsing Hua University, Hsinchu 30013, Taiwan \\ ${ }^{3}$ Department of Electronic Engineering, University of Tor Vergata, Via del Politecnico 1, 00133 Rome, Italy \\ ${ }^{4}$ Department of Engineering, University of Cambridge, $9 \mathrm{JJ}$ Thomson Avenue, Cambridge CB3 OFA, UK
}

Correspondence should be addressed to Wenjie Mai; wenjiemai@gmail.com

Received 3 June 2014; Accepted 3 June 2014; Published 18 June 2014

Copyright ( 92014 Wenjie Mai et al. This is an open access article distributed under the Creative Commons Attribution License, which permits unrestricted use, distribution, and reproduction in any medium, provided the original work is properly cited.

Energy generation and storage are perhaps two of the most imperative, ongoing, and challenging issues facing the world to date. The rapidly growing global energy demand is placing increasingly greater burdens on the incumbent fossilfuel based infrastructure necessitating the development and mobilization of alternative technologies, particularly those focused on exploiting or scavenging new and clean energy sources alongside the development of highly efficient energy storage devices. Though advanced energy storage concepts and regenerative energy systems have been the focus of considerable attention over the past decade, the advent of the nanoage added traction to field by promising the potential to fabricate, for the first time, a wide range of truly novel devices that will enable this paradigm shift in energy applications. However, breakthroughs are needed to synthesize, engineer, and manufacture, en masse, nanomaterials and micro- and macrostructures based thereon. Central to this is a new understanding of the atomistic assembly of such materials in order to study energy transduction at nanoscale and to elucidate the underlying chemical and physical mechanism involved in high efficient energy conversion and storage.

This special issue-which focuses on the science and engineering of nanomaterials and nanodevices used in all forms of energy harvesting, conversion, storage, and utilization-consists of 13 papers spanning topics detailing the empirical exploitation of nanowires for enhanced supercapacitors to thermoelectric generators, photocatalytic nanoparticles, and small molecule based organic solar cells. L. Chen et al. discuss the use of scanning force microscopy to investigate frictional images of newly cleaved surface domains of single crystal $\mathrm{BaTiO}_{3}$ under ambipolar biases, providing a new method to determine domain polarization direction, whereas H.-S. Tsai et al. synthesized novel onedimensional $\mathrm{SiO}_{2} / \mathrm{Ta}_{2} \mathrm{O}_{5}$ core-shell nanostructures, proposing a Ta-O diffusion-controlled growth process. P. Hiralal et al. report on the tailored growth of carbon nanostructures for application in high-frequency supercapacitors formed from engineered vertically aligned nanocarbon electrodes which are shown to enhance the porosity and electrolyte permeability within the device, resulting in a novel fabrication route to realise a functionally advanced compact energy storage platform. In a similar approach, $\mathrm{H}$. Xu et al. summarise their work towards the preparation of $\mathrm{Co}_{3} \mathrm{O}_{4}$ nanoparticles which facilitate long-lifetime and electrochemically superior supercapacitors by undertaking detailed studies of the electrode metrology. Z. Ma et al. report on energy recovery of coolant heat for internal combustion engines using thermoelectric generation technology. They showed that cooling improved with increased fan speeds and that the Seebeck coefficient was reduced with an increase in temperature difference between the two ends of thermoelectric generator.

\section{Acknowledgment}

We would like to express our sincerest gratitude to all the authors and reviewers who kindly contributed their time to this special issue. We hope that the wider readership finds this special edition accurate, insightful, and a pragmatic guide to the state-of-the art nanobased energy applications.

Wenjie Mai Yu-Lun Chueh Christian Falconi Matthew T. Cole 

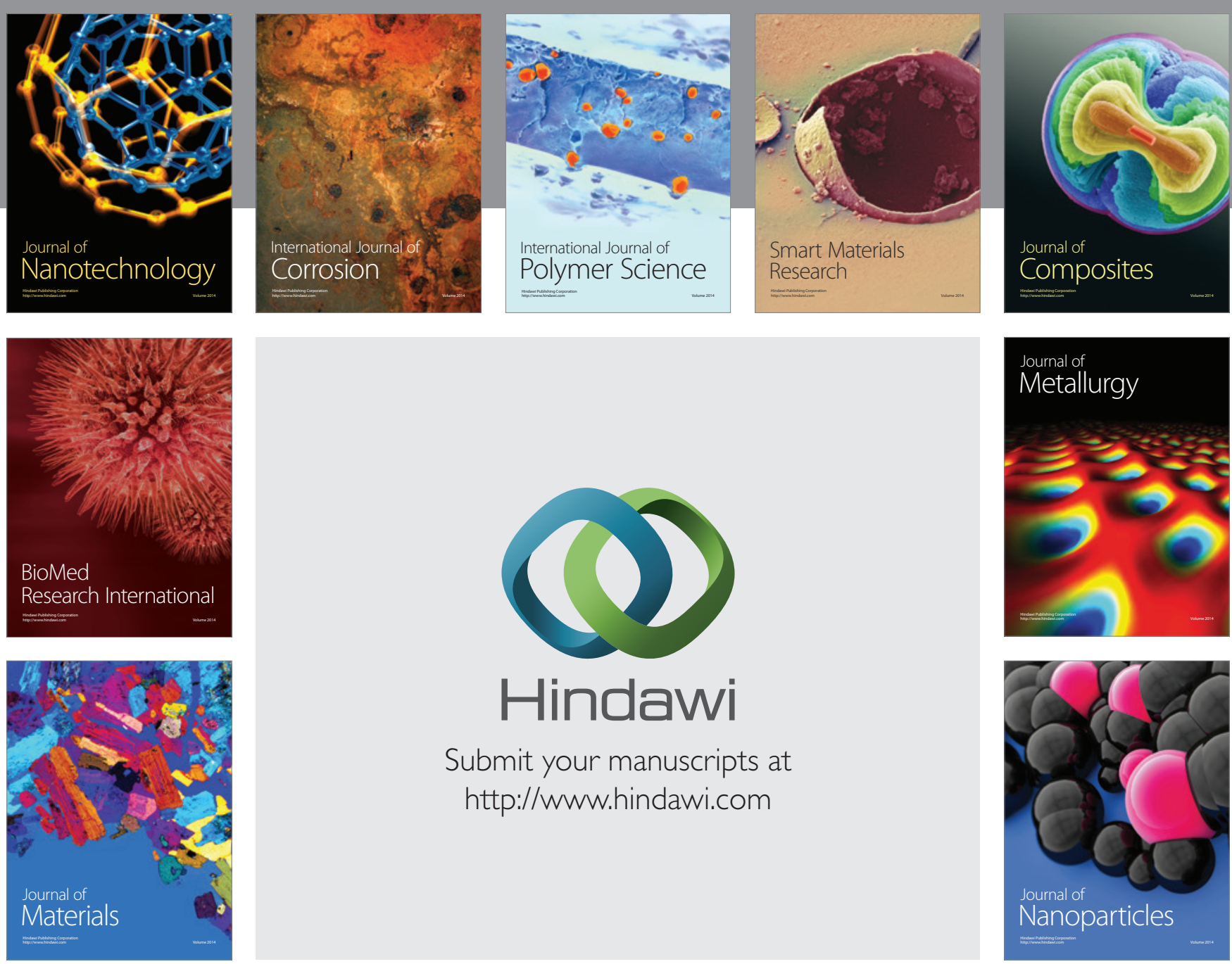

Submit your manuscripts at http://www.hindawi.com
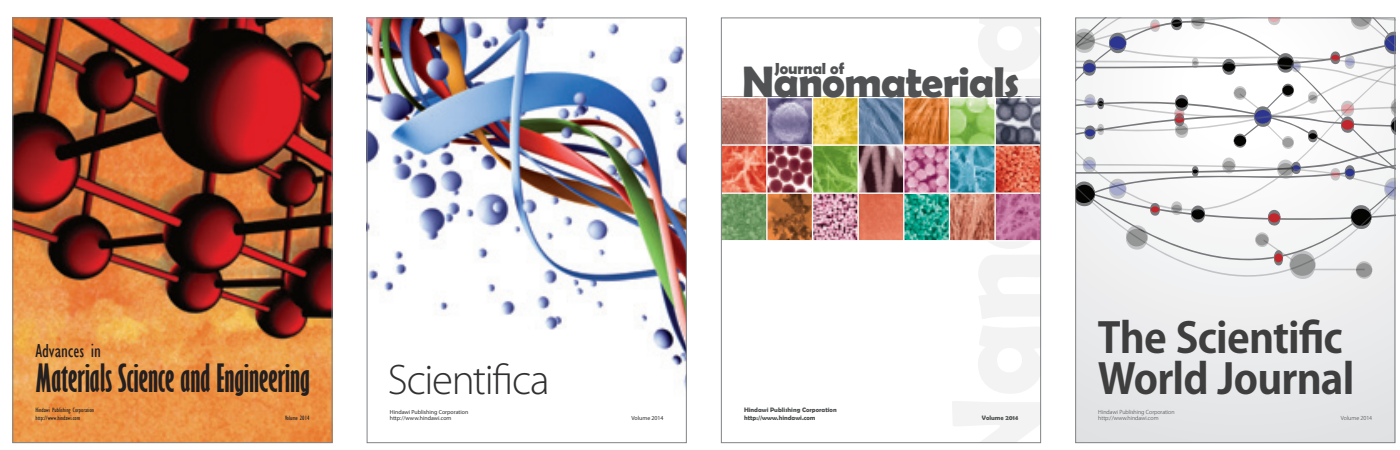

\section{The Scientific World Journal}
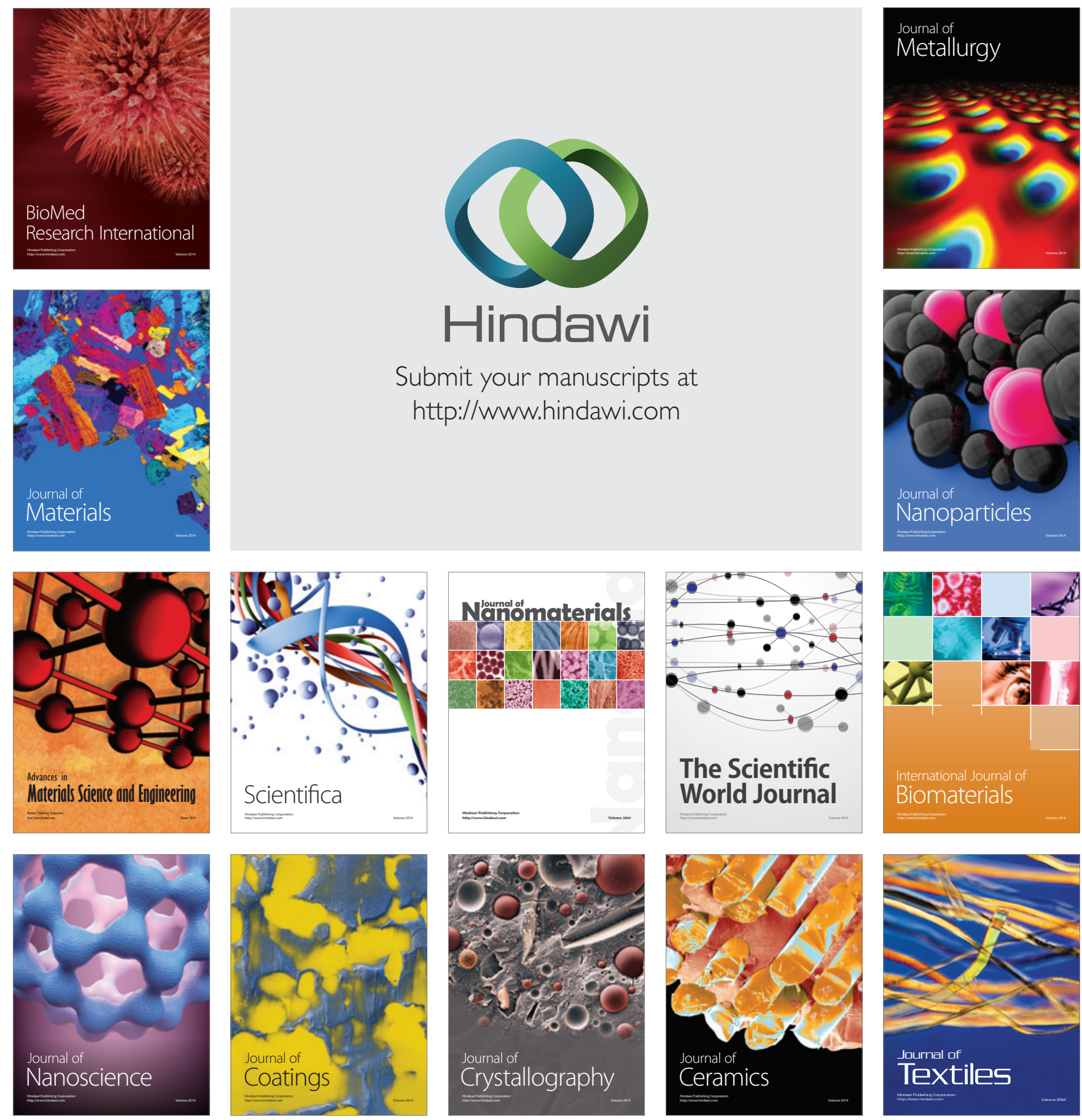